\title{
A Discrete Dirac-Kähler Equation Using a Geometric Discretisation Scheme
}

\author{
Volodymyr Sushch*0
}

\author{
Communicated by Uwe Kaehler
}

\begin{abstract}
Discrete models of the Dirac-Kähler equation and the Dirac equation in the Hestenes form are discussed. A discrete version of the plane wave solutions to a discrete analogue of the Hestenes equation is established.
\end{abstract}

Mathematics Subject Classification. Primary 39A12; Secondary 81Q05, 39A70.

Keywords. Dirac-Kähler equation, Hestenes equation, Discrete models, Clifford multiplication, Plane wave solution.

\section{Introduction}

We study a discrete model of the Dirac-Kähler equation in which key geometric aspects of the continuum counterpart are captured. We pay special attention to the description of some method of construction a discretisation scheme based on the use of the differential forms calculus. The aim of this paper is to establish some discrete version of the plane wave solutions to a discrete Dirac equation in the Hestenes form. To construct these discrete solutions we introduce a Clifford product acting on the space of discrete inhomogeneous forms. This work is a direct continuation of that described in my previous papers [12-15]. In [15], a correspondence between a discrete Dirac-Kähler equation and a discrete analogue of the Hestenes equation was studied. There are several approaches to study of discrete versions of the Dirac-Kähler equation based on the use a discrete Clifford calculus framework on lattices. For a review of discrete Clifford analysis, we refer the reader to $[4-6,10,16]$. It is beyond the scope of this paper to fully discuss differences and intersections between approaches.

We first briefly review some definitions and basic facts on the DiracKähler equation $[9,11]$ and the Dirac equation in the spacetime algebra $[7,8]$.

${ }^{*}$ Corresponding author. 
Let $M=\mathbb{R}^{1,3}$ be Minkowski space with metric signature $(+,-,-,-)$. Denote by $\Lambda^{r}(M)$ the vector space of smooth differential $r$-forms, $r=0,1,2,3,4$. We consider $\Lambda^{r}(M)$ over $\mathbb{C}$. Let $\omega, \varphi \in \Lambda^{r}(M)$. The inner product is defined by

$$
(\omega, \varphi)=\int_{M} \omega \wedge * \bar{\varphi},
$$

where $\Lambda$ is the exterior product and $*$ is the Hodge star operator $*: \Lambda^{r}(M) \rightarrow$ $\Lambda^{4-r}(M)$ with respect to the Lorentz metric. Let $d: \Lambda^{r}(M) \rightarrow \Lambda^{r+1}(M)$ be the exterior differential and let $\delta: \Lambda^{r}(M) \rightarrow \Lambda^{r-1}(M)$ be the formal adjoint of $d$ with respect to (1.1). We have

$$
\delta=* d * .
$$

Denote by $\Lambda(M)$ the set of all differential forms on $M$. We have

$$
\Lambda(M)=\Lambda^{e v}(M) \oplus \Lambda^{o d}(M),
$$

where $\Lambda^{e v}(M)=\Lambda^{0}(M) \oplus \Lambda^{2}(M) \oplus \Lambda^{4}(M)$ and $\Lambda^{\text {od }}(M)=\Lambda^{1}(M) \oplus \Lambda^{3}(M)$. Let $\Omega \in \Lambda(M)$ be an inhomogeneous differential form, i.e.,

$$
\Omega=\sum_{r=0}^{4} \stackrel{r}{\omega}
$$

where $\stackrel{r}{\omega} \in \Lambda^{r}(M)$. The Dirac-Kähler equation for a free electron is given by

$$
i(d+\delta) \Omega=m \Omega
$$

where $i$ is the usual complex unit and $m$ is a mass parameter. It is easy to show that Eq. (1.2) is equivalent to the set of equations

$$
\begin{aligned}
& i \delta \stackrel{1}{\omega}=m \stackrel{0}{\omega}, \\
& i\left(d \omega{ }^{0}+\delta \stackrel{2}{\omega}\right)=m \stackrel{1}{\omega}, \\
& i(d \stackrel{1}{\omega}+\delta \stackrel{3}{\omega})=m \stackrel{2}{\omega}, \\
& i(d \stackrel{2}{\omega}+\delta \stackrel{4}{\omega})=m \stackrel{3}{\omega}, \\
& i d \stackrel{3}{\omega}=m \stackrel{4}{\omega} .
\end{aligned}
$$

Denote by $\Omega^{e v}$ and by $\Omega^{o d}$ the even and odd parts of $\Omega$. It is clear that

$$
\begin{aligned}
& i(d+\delta) \Omega^{o d}=m \Omega^{e v}, \\
& i(d+\delta) \Omega^{e v}=m \Omega^{o d} .
\end{aligned}
$$

The operator $d+\delta$ is the analogue of the gradient operator in Minkowski spacetime

$$
\nabla=\sum_{\mu=0}^{3} \gamma_{\mu} \partial^{\mu}, \quad \mu=0,1,2,3
$$

where $\gamma_{\mu}$ is the Dirac gamma matrix. If one think of $\left\{\gamma_{0}, \gamma_{1}, \gamma_{2}, \gamma_{3}\right\}$ as a vector basis in spacetime, then the gamma matrices $\gamma_{\mu}$ can be considered as generators of the Clifford algebra of spacetime $\mathcal{C} \ell(1,3)[1,2]$. Hestenes [8] calls this algebra the spacetime algebra. Denote by $C \ell_{\mathbb{R}}(1,3)\left(C \ell_{\mathbb{C}}(1,3)\right)$ the 
real (complex) Clifford algebra. It is known that an inhomogeneous form $\Omega$ can be represented as element of $\mathcal{C} \ell_{\mathbb{C}}(1,3)$. Then the Dirac-Kähler equation can be written as the algebraic equation

$$
i \nabla \Omega=m \Omega, \quad \Omega \in C \ell_{\mathbb{C}}(1,3) .
$$

Equation (1.4) is equivalent to the four Dirac equations (traditional columnspinor equations) for a free electron. Let $C \ell^{e v}(1,3)$ be the even subalgebra of the algebra $C \ell(1,3)$. The equation

$$
-\nabla \Omega^{e v} \gamma_{1} \gamma_{2}=m \Omega^{e v} \gamma_{0}, \quad \Omega^{e v} \in C \ell_{\mathbb{R}}^{e v}(1,3),
$$

is called the Hestenes form of the Dirac equation $[7,8]$.

It should be noted that the graded algebra $\Lambda(M)$ endowed with the Clifford multiplication is an example of a Clifford algebra. In this case the basis covectors $e^{\mu}=d x^{\mu}$ are considered as generators of the Clifford algebra. Then the Hestenes equation (1.5) can be rewritten in terms of inhomogeneous forms as

$$
-(d+\delta) \Omega^{e v} e^{1} e^{2}=m \Omega^{e v} e^{0}, \quad \Omega^{e v} \in \Lambda_{\mathbb{R}}^{e v}(M) .
$$

\section{Discretisation Scheme}

The starting point for consideration is a combinatorial model of Euclidean space. The proposed approach was originated by Dezin [3]. For the convenience of the reader we briefly repeat the relevant material from [13] without proofs, thus making our presentation self-contained.

Let the tensor product

$$
C(4)=C \otimes C \otimes C \otimes C,
$$

of a 1-dimensional complex be a combinatorial model of Euclidean space $\mathbb{R}^{4}$. The 1-dimensional complex $C$ is defined in the following way. Introduce the sets $\left\{x_{\kappa}\right\}$ and $\left\{e_{\kappa}\right\}, \kappa \in \mathbb{Z}$. Let $C^{0}$ and $C^{1}$ be the free abelian groups of 0 -dimensional and 1-dimensional chains generated by $\left\{x_{\kappa}\right\}$ and $\left\{e_{\kappa}\right\}$. The free abelian group is understood as the direct sum of infinity cyclic groups generated by $\left\{x_{\kappa}\right\},\left\{e_{\kappa}\right\}$. Arbitrary elements (chains) $a \in C^{0}$ and $b \in C^{1}$ can be written as the formal sums

$$
a=\sum_{\kappa} a^{\kappa} x_{\kappa}, \quad b=\sum_{\kappa} b^{\kappa} e_{\kappa}, \quad a^{\kappa}, b^{\kappa} \in \mathbb{Z} .
$$

We define the boundary operator $\partial: C^{1} \rightarrow C^{0}, \quad \partial: C^{0} \rightarrow 0$, setting

$$
\partial e_{\kappa}=x_{\kappa+1}-x_{\kappa}, \quad \partial x_{\kappa}=0 .
$$

The definition (2.1) is extended to arbitrary chains by linearity.

The direct sum $C=C^{0} \oplus C^{1}$ with the boundary operator $\partial$ defines the 1 dimensional complex. It is known that a free abelian group is an abelian group with basis. One can regard the sets $\left\{x_{\kappa}\right\},\left\{e_{\kappa}\right\}$ as sets of basis elements of the groups $C^{0}$ and $C^{1}$. Geometrically we can interpret the 0 -dimensional basis elements $x_{\kappa}$ as points of the real line and the 1-dimensional basis elements $e_{\kappa}$ as open intervals between points. We call the complex $C$ a combinatorial real line. 
Multiplying the basis elements $x_{\kappa}, e_{\kappa}$ in various way we obtain basis elements of $C(4)$. Let $s_{k}^{(r)}$ be an arbitrary $r$-dimensional basis element of $C(4)$. Then we have

$$
s_{k}^{(r)}=s_{k_{0}} \otimes s_{k_{1}} \otimes s_{k_{2}} \otimes s_{k_{3}},
$$

where $s_{k_{\mu}}$ is either $x_{k_{\mu}}$ or $e_{k_{\mu}}$ and $k=\left(k_{0}, k_{1}, k_{2}, k_{3}\right), k_{\mu} \in \mathbb{Z}$. The dimension $r$ of a basis element $s_{k}^{(r)}$ is given by the number of factors $e_{k_{\mu}}$ that appear in it. The product contains exactly $r$ 1-dimensional elements $e_{k_{\mu}}$ and $4-r$ 0-dimensional elements $x_{k_{\mu}}$, and the superscript $(r)$ indicates also a position of $e_{k_{\mu}}$ in $s_{k}^{(r)}$. For example, the 1- and 2-dimensional basis elements of $C(4)$ can be written as

$$
\begin{aligned}
& e_{k}^{0}=e_{k_{0}} \otimes x_{k_{1}} \otimes x_{k_{2}} \otimes x_{k_{3}}, \quad e_{k}^{1}=x_{k_{0}} \otimes e_{k_{1}} \otimes x_{k_{2}} \otimes x_{k_{3}}, \\
& e_{k}^{2}=x_{k_{0}} \otimes x_{k_{1}} \otimes e_{k_{2}} \otimes x_{k_{3}}, \quad e_{k}^{3}=x_{k_{0}} \otimes x_{k_{1}} \otimes x_{k_{2}} \otimes e_{k_{3}}
\end{aligned}
$$

and

$$
\begin{array}{ll}
e_{k}^{01}=e_{k_{0}} \otimes e_{k_{1}} \otimes x_{k_{2}} \otimes x_{k_{3}}, & e_{k}^{12}=x_{k_{0}} \otimes e_{k_{1}} \otimes e_{k_{2}} \otimes x_{k_{3}}, \\
e_{k}^{02}=e_{k_{0}} \otimes x_{k_{1}} \otimes e_{k_{2}} \otimes x_{k_{3}}, & e_{k}^{13}=x_{k_{0}} \otimes e_{k_{1}} \otimes x_{k_{2}} \otimes e_{k_{3}}, \\
e_{k}^{03}=e_{k_{0}} \otimes x_{k_{1}} \otimes x_{k_{2}} \otimes e_{k_{3}}, & e_{k}^{23}=x_{k_{0}} \otimes x_{k_{1}} \otimes e_{k_{2}} \otimes e_{k_{3}} .
\end{array}
$$

Let $C(4)=C(p) \otimes C(q)$, where $p+q=4$ and $C(p)$ is the tensor product of $p$ factors of $C=C(1), p=1,2,3$. The definition (2.1) of $\partial$ is extended to arbitrary chains of $C(4)$ by the rule

$$
\partial(a \otimes b)=\partial a \otimes b+(-1)^{r} a \otimes \partial b,
$$

where $a \in C(p)$ and $b \in C(q)$ and $r$ is the dimension of the chain $a$. It is easy to check that $\partial \partial a=0$ for any $a \in C(4)$.

Suppose that the combinatorial model of Minkowski space has the same structure as $C(4)$. We will use the index $k_{0}$ to denote the basis elements of $C$ which correspond to the time coordinate of $M$. Hence, the indicated basis elements will be written as $x_{k_{0}}, e_{k_{0}}$.

Let us introduce the construction of a double complex. This construction generalizes [13]. Together with the complex $C(4)$ we consider its double, namely the complex $\tilde{C}(4)$ of exactly the same structure, i.e., the one in the corresponding chains $a \in C(4)$ and $\tilde{a} \in \tilde{C}(4)$ have the same coefficients. Define the one-to-one correspondence

$$
*^{c}: C(4) \rightarrow \tilde{C}(4), \quad *^{c}: \tilde{C}(4) \rightarrow C(4)
$$

by the rule

where

$$
*^{c} s_{k}^{(r)}=\varepsilon(r) \tilde{s}_{k}^{(4-r)}, \quad *^{c} \tilde{s}_{k}^{(r)}=\varepsilon(r) s_{k}^{(4-r)},
$$

$$
\tilde{s}_{k}^{(4-r)}=*^{c} s_{k_{0}} \otimes *^{c} s_{k_{1}} \otimes *^{c} s_{k_{2}} \otimes *^{c} s_{k_{3}}
$$

and $*^{c} x_{k_{\mu}}=\tilde{e}_{k_{\mu}}, *^{c} e_{k_{\mu}}=\tilde{x}_{k_{\mu}}$. Here $\varepsilon(r)$ is the Levi-Civita symbol, i.e.,

$$
\varepsilon(r)=\left\{\begin{array}{lll}
+1 & \text { if } \quad((r),(4-r)) & \text { is an even permutation of }(0,1,2,3) \\
-1 & \text { if } \quad((r),(4-r)) & \text { is an odd permutation of }(0,1,2,3) .
\end{array}\right.
$$


For example, for the 1- and 2-dimensional basis elements we have

$$
*^{c} e_{k}^{0}=\tilde{e}_{k}^{123}, \quad *^{c} e_{k}^{1}=-\tilde{e}_{k}^{023}, \quad *^{c} e_{k}^{2}=\tilde{e}_{k}^{013}, \quad *^{c} e_{k}^{3}=-\tilde{e}_{k}^{012}
$$

and

$$
\begin{array}{lll}
*^{c} e_{k}^{01}=\tilde{e}_{k}^{23}, & *^{c} e_{k}^{02}=-\tilde{e}_{k}^{13}, & *^{c} e_{k}^{03}=\tilde{e}_{k}^{12}, \\
*^{c} e_{k}^{12}=\tilde{e}_{k}^{03}, & *^{c} e_{k}^{13}=-\tilde{e}_{k}^{02}, & *^{c} e_{k}^{23}=\tilde{e}_{k}^{01} .
\end{array}
$$

The operation (2.3) is linearly extended to chains.

Proposition 2.1. Let $a_{r} \in C(4)$ be an $r$-dimensional chain, then we have

$$
*^{c} *^{c} a_{r}=(-1)^{r} a_{r} .
$$

Proof. The proof consists in applying the operation $*^{c}$ for basis elements.

Let us now consider a dual complex to $C(4)$. We define it as the complex of cochains $K(4)$ with complex coefficients. The complex $K(4)$ has a similar structure, namely $K(4)=K \otimes K \otimes K \otimes K$, where $K$ is a dual complex to the 1-dimensional complex $C$. Let $x^{\kappa}$ and $e^{\kappa}, \kappa \in \mathbb{Z}$, be the 0 - and 1-dimensional basis elements of $K$. Then an arbitrary $r$-dimensional basis element of $K(4)$ can be written as $s_{(r)}^{k}=s^{k_{0}} \otimes s^{k_{1}} \otimes s^{k_{2}} \otimes s^{k_{3}}$, where $s^{k_{\mu}}$ is either $x^{k_{\mu}}$ or $e^{k_{\mu}}$ and $k=\left(k_{0}, k_{1}, k_{2}, k_{3}\right)$. We will call cochains forms, emphasizing their relationship with differential forms. Denote by $K^{r}(4)$ the set of all $r$-forms. Then $K(4)$ can be expressed by

$$
K(4)=K^{e v}(4) \oplus K^{o d}(4),
$$

where $K^{e v}(4)=K^{0}(4) \oplus K^{2}(4) \oplus K^{4}(4)$ and $K^{o d}(4)=K^{1}(4) \oplus K^{3}(4)$. The complex $K(4)$ is a discrete analogue of $\Lambda(M)$. Let $\stackrel{r}{\omega} \in K^{r}(4)$, then we have

$$
\begin{aligned}
& \stackrel{0}{\omega}=\sum_{k} \stackrel{0}{\omega}_{k} x^{k}, \quad \stackrel{2}{\omega}=\sum_{k} \sum_{\mu<\nu} \omega_{k}^{\mu \nu} e_{\mu \nu}^{k}, \quad \stackrel{4}{\omega}=\sum_{k} \stackrel{4}{\omega}_{k} e^{k}, \\
& \stackrel{1}{\omega}=\sum_{k} \sum_{\mu=0}^{3} \omega_{k}^{\mu} e_{\mu}^{k}, \quad \stackrel{3}{\omega}=\sum_{k} \sum_{\iota<\mu<\nu} \omega_{k}^{\iota \mu \nu} e_{\iota \mu \nu}^{k},
\end{aligned}
$$

where $e_{\mu}^{k}, e_{\mu \nu}^{k}$ and $e_{\iota \mu \nu}^{k}$ are the 1-, 2- and 3-dimensional basis elements of $K(4)$, and $x^{k}=x^{k_{0}} \otimes x^{k_{1}} \otimes x^{k_{2}} \otimes x^{k_{3}}, \quad e^{k}=e^{k_{0}} \otimes e^{k_{1}} \otimes e^{k_{2}} \otimes e^{k_{3}}$. The components $\stackrel{0}{\omega}_{k}, \stackrel{4}{\omega}_{k}, \omega_{k}^{\mu}, \omega_{k}^{\mu \nu}$ and $\omega_{k}^{\iota \mu \nu}$ are complex numbers.

We define the pairing (chain-cochain) operation for any basis elements $\epsilon_{k} \in C(4), s^{k} \in K(4)$ by the rule

$$
\left\langle\epsilon_{k}, s^{k}\right\rangle= \begin{cases}0, & \epsilon_{k} \neq s_{k} \\ 1, & \epsilon_{k}=s_{k} .\end{cases}
$$

The operation (2.4) is linearly extended to arbitrary chains-cochains.

The coboundary operator $d^{c}: K^{r}(4) \rightarrow K^{r+1}(4)$ is defined by

$$
\left\langle\partial a_{r+1}, \stackrel{r}{\omega}\right\rangle=\left\langle a_{r+1}, d^{c} \stackrel{r}{\omega}\right\rangle,
$$


where $a_{r+1} \in C(4)$ is an $r+1$ dimensional chain. The operator $d^{c}$ is an analog of the exterior differential. From the above it follows that

$$
d^{c} \stackrel{4}{\omega}=0 \text { and } \quad d^{c} d^{c} \stackrel{r}{\omega}=0 \text { for any } r
$$

Let the difference operator $\Delta_{\mu}$ be defined by

$$
\Delta_{\mu} \omega_{k}^{(r)}=\omega_{\tau_{\mu} k}^{(r)}-\omega_{k}^{(r)},
$$

where $\omega_{k}^{(r)} \in \mathbb{C}$ is a component of $\stackrel{r}{\omega} \in K^{r}(4)$ and $\tau_{\mu}$ is the shift operator which acts as

$$
\tau_{\mu} k=\left(k_{0}, \ldots k_{\mu}+1, \ldots k_{3}\right), \quad \mu=0,1,2,3 .
$$

Using (2.2) and (2.5) we can calculate

$$
\begin{aligned}
& d^{c} \stackrel{0}{\omega}=\sum_{k} \sum_{\mu=0}^{3}\left(\Delta_{\mu} \stackrel{0}{\omega}_{k}\right) e_{\mu}^{k}, \quad d^{c} \stackrel{1}{\omega}=\sum_{k} \sum_{\mu<\nu}\left(\Delta_{\mu} \omega_{k}^{\nu}-\Delta_{\nu} \omega_{k}^{\mu}\right) e_{\mu \nu}^{k} \\
& d^{c} \stackrel{2}{\omega}=\sum_{k} {\left[\left(\Delta_{0} \omega_{k}^{12}-\Delta_{1} \omega_{k}^{02}+\Delta_{2} \omega_{k}^{01}\right) e_{012}^{k}\right.} \\
&+\left(\Delta_{0} \omega_{k}^{13}-\Delta_{1} \omega_{k}^{03}+\Delta_{3} \omega_{k}^{01}\right) e_{013}^{k} \\
&+\left(\Delta_{0} \omega_{k}^{23}-\Delta_{2} \omega_{k}^{03}+\Delta_{3} \omega_{k}^{02}\right) e_{023}^{k} \\
&+\left.\left(\Delta_{1} \omega_{k}^{23}-\Delta_{2} \omega_{k}^{13}+\Delta_{3} \omega_{k}^{12}\right) e_{123}^{k}\right] \\
& d^{c} \stackrel{3}{\omega}=\sum_{k}\left(\Delta_{0} \omega_{k}^{123}-\Delta_{1} \omega_{k}^{023}+\Delta_{2} \omega_{k}^{013}-\Delta_{3} \omega_{k}^{012}\right) e^{k}
\end{aligned}
$$

Let $\tilde{K}(4)$ be a complex of the cochains over the double complex $\tilde{C}(4)$, with the coboundary operator $d^{c}$ defined by (2.5). Hence, $\tilde{K}(4)$ has the same structure as $K(4)$. This means that the corresponding forms $\omega \in K(4)$ and $\tilde{\omega} \in \tilde{K}(4)$ have the same components.

Let us introduce a discrete version of the Hodge star operator by using the double complex construction. Define the operation $*: K^{r}(4) \rightarrow \tilde{K}^{4-r}(4)$ for the basis element $s_{(r)}^{k} \in K^{r}(4)$ by the rule

$$
* s_{(r)}^{k}=Q\left(k_{0}\right) \varepsilon(r) \tilde{s}_{(4-r)}^{k},
$$

where

$$
Q\left(k_{0}\right)=\left\{\begin{array}{lll}
+1 & \text { if } \quad s^{k_{0}}=x^{k_{0}} \\
-1 & \text { if } \quad s^{k_{0}}=e^{k_{0}} .
\end{array}\right.
$$

This definition makes sense because the formula (2.10) preserves the Lorentz signature of metric on $K(4)$. More explicitly, we have

$$
\begin{aligned}
& * x^{k}=\tilde{e}^{k}, \quad * e^{k}=-\tilde{x}^{k}, \\
& * e_{0}^{k}=-\tilde{e}_{123}^{k}, \quad * e_{1}^{k}=-\tilde{e}_{023}^{k}, \quad * e_{2}^{k}=\tilde{e}_{013}^{k}, \quad * e_{3}^{k}=-\tilde{e}_{012}^{k}, \\
& * e_{01}^{k}=-\tilde{e}_{23}^{k}, \quad * e_{02}^{k}=\tilde{e}_{13}^{k}, \quad * e_{03}^{k}=-\tilde{e}_{12}^{k}, \\
& * e_{12}^{k}=\tilde{e}_{03}^{k}, \quad * e_{13}^{k}=-\tilde{e}_{02}^{k}, \quad * e_{23}^{k}=\tilde{e}_{01}^{k}, \\
& * e_{012}^{k}=-\tilde{e}_{3}^{k}, \quad * e_{013}^{k}=\tilde{e}_{2}^{k}, \quad * e_{023}^{k}=-\tilde{e}_{1}^{k}, \quad * e_{123}^{k}=-\tilde{e}_{0}^{k} .
\end{aligned}
$$


For any $r$-form the operation (2.10) is extended by linearity. Likewise, the mapping $*: \tilde{K}^{r}(4) \rightarrow K^{4-r}(4)$ is given by the rule $(2.10)$.

Proposition 2.2. Let $\stackrel{r}{\omega} \in K^{r}(4)$, then we have

$$
* * \stackrel{r}{\omega}=(-1)^{r+1} \stackrel{r}{\omega} .
$$

Proof. The operation $*$ is linear. It is easy to check that by definition, the composition of $*$ with itself gives

$$
* * s_{(r)}^{k}=(-1)^{r(4-r)+1} s_{(r)}^{k}=(-1)^{r+1} s_{(r)}^{k},
$$

for any basis element $s_{(r)}^{k} \in K^{r}(4)$.

Hence, the operation $*^{2}$ is either an involution or antiinvolution. It means that the discrete $*$ operation imitates correctly the continuum case.

Proposition 2.3. Let $\tilde{a}_{r} \in \tilde{C}(4)$ be an $r$-dimensional chain and let $\omega \in$ $K^{4-r}(4)$. Then we have

$$
\left\langle\tilde{a}_{r}, * \omega\right\rangle=(-1)^{r} Q\left(k_{0}\right)\left\langle *^{c} \tilde{a}_{r}, \omega\right\rangle .
$$

Proof. See Proposition 3 in [13].

Let us consider the 4-dimensional finite chain $e_{n} \subset C(4)$ of the form:

$$
e_{n}=\sum_{k} e_{k}, \quad k=\left(k_{0}, k_{1}, k_{2}, k_{3}\right), \quad k_{\mu}=1,2, \ldots, n_{\mu},
$$

where $n_{\mu} \in \mathbb{N}$ is a fixed number for each $\mu=0,1,2,3$. This finite sum of 4-dimensional basis elements of $C(4)$ imitates a domain of $M$. We set

$$
V_{r}=\sum_{k} \sum_{(r)} s_{k}^{(r)} \otimes *{ }^{c} s_{k}^{(r)}, \quad k_{\mu}=1,2, \ldots, n_{\mu} .
$$

For example,

$$
V_{1}=\sum_{k} \sum_{\mu=0}^{3} e_{k}^{\mu} \otimes *^{c} e_{k}^{\mu}=\sum_{k}\left(e_{k}^{0} \otimes \tilde{e}_{k}^{123}-e_{k}^{1} \otimes \tilde{e}_{k}^{023}+e_{k}^{2} \otimes \tilde{e}_{k}^{013}-e_{k}^{3} \otimes \tilde{e}_{k}^{012}\right) .
$$

Let

$$
\mathbb{V}=\sum_{r=0}^{4} V_{r}
$$

For any $r$-forms $\varphi, \omega \in K^{r}(4)$ the inner product over the set $e_{n}(2.12)$ is defined by the rule

$$
(\varphi, \omega)_{e_{n}}=\langle\mathbb{V}, \varphi \otimes * \bar{\omega}\rangle
$$

where $\bar{\omega}$ denotes the complex conjugate of the form $\omega$. For forms of different degrees the product (2.13) is set equal to zero. It is clear that

$$
(\varphi, \omega)_{e_{n}}=\left\langle V_{r}, \varphi \otimes * \bar{\omega}\right\rangle=\sum_{k} \sum_{(r)}\left\langle s_{k}^{(r)}, \varphi\right\rangle\left\langle *^{c} s_{k}^{(r)}, * \bar{\omega}\right\rangle .
$$

For example, if $\stackrel{1}{\varphi}, \stackrel{1}{\omega} \in K^{1}(4)$ then we obtain

$$
(\stackrel{1}{\varphi}, \stackrel{1}{\omega})_{e_{n}}=\sum_{k}\left(-\varphi_{k}^{0} \bar{\omega}_{k}^{0}+\varphi_{k}^{1} \bar{\omega}_{k}^{1}+\varphi_{k}^{2} \bar{\omega}_{k}^{2}+\varphi_{k}^{3} \bar{\omega}_{k}^{3}\right)
$$


It should be noted that the definition of the inner product correctly imitates the case (1.1) and the Lorentz metric structure is still captured here.

The inner product (2.13) makes it possible to define the adjoint of $d^{c}$, denoted $\delta^{c}$.

Proposition 2.4. For any $(r-1)$-form $\varphi$ and $r$-form $\omega$ we have

$$
\left(d^{c} \varphi, \omega\right)_{e_{n}}=\langle\partial \mathbb{V}, \varphi \otimes * \bar{\omega}\rangle+\left(\varphi, \delta^{c} \omega\right)_{e_{n}},
$$

where

$$
\delta^{c}=(-1)^{r} *^{-1} d^{c} *,
$$

and $*^{-1}$ is the inverse operation of $*$.

Proof. The proof is a direct computation. See Proposition 4 in [13].

The relation (2.14) is a discrete analog of the Green formula. From (2.11) we infer

$$
*^{-1}=(-1)^{r(4-r)+1} *=(-1)^{r+1} * .
$$

Putting this in (2.15) we obtain

$$
\delta^{c}=* d^{c} * .
$$

This makes it clear that the operator $\delta^{c}: K^{r+1}(4) \rightarrow K^{r}(4)$ is a discrete analog of the codifferential $\delta$. From (2.16) it follows that

$$
\delta^{c} \stackrel{0}{\omega}=0 \text { and } \quad \delta^{c} \delta^{c} \stackrel{r}{\omega}=0 \text { for any } r .
$$

Using the definitions of $d^{c}$ and $*$ we can calculate

$$
\begin{aligned}
\delta^{c} \stackrel{1}{\omega}= & \sum_{k}\left(\Delta_{0} \omega_{k}^{0}-\Delta_{1} \omega_{k}^{1}-\Delta_{2} \omega_{k}^{2}-\Delta_{3} \omega_{k}^{3}\right) x^{k} \\
\delta^{c} \stackrel{2}{\omega}= & \sum_{k}\left[\left(\Delta_{1} \omega_{k}^{01}+\Delta_{2} \omega_{k}^{02}+\Delta_{3} \omega_{k}^{03}\right) e_{0}^{k}\right. \\
& +\left(\Delta_{0} \omega_{k}^{01}+\Delta_{2} \omega_{k}^{12}+\Delta_{3} \omega_{k}^{13}\right) e_{1}^{k} \\
& +\left(\Delta_{0} \omega_{k}^{02}-\Delta_{1} \omega_{k}^{12}+\Delta_{3} \omega_{k}^{23}\right) e_{2}^{k} \\
& \left.+\left(\Delta_{0} \omega_{k}^{03}-\Delta_{1} \omega_{k}^{13}-\Delta_{2} \omega_{k}^{23}\right) e_{3}^{k}\right] \\
\delta^{c} \stackrel{3}{\omega}= & \sum_{k}\left[\left(-\Delta_{2} \omega_{k}^{012}-\Delta_{3} \omega_{k}^{013}\right) e_{01}^{k}+\left(\Delta_{1} \omega_{k}^{012}-\Delta_{3} \omega_{k}^{023}\right) e_{02}^{k}\right. \\
& +\left(\Delta_{1} \omega_{k}^{013}+\Delta_{2} \omega_{k}^{023}\right) e_{03}^{k}+\left(\Delta_{0} \omega_{k}^{012}-\Delta_{3} \omega_{k}^{123}\right) e_{12}^{k} \\
& \left.+\left(\Delta_{0} \omega_{k}^{013}+\Delta_{2} \omega_{k}^{123}\right) e_{13}^{k}+\left(\Delta_{0} \omega_{k}^{023}-\Delta_{1} \omega_{k}^{123}\right) e_{23}^{k}\right] \\
\delta^{c} \stackrel{4}{\omega}= & \sum_{k}\left[\left(\Delta_{3} \omega_{k}\right) e_{012}^{k}-\left(\Delta_{2} \omega_{k}\right) e_{013}^{k}+\left(\Delta_{1} \omega_{k}\right) e_{023}^{k}+\left(\Delta_{0} \omega_{k}\right) e_{123}^{k}\right]
\end{aligned}
$$

The linear map

$$
\Delta^{c}=-\left(d^{c} \delta^{c}+\delta^{c} d^{c}\right): K^{r}(4) \rightarrow K^{r}(4),
$$

is called a discrete analogue of the Laplacian. It is clear that

$$
-\left(d^{c} \delta^{c}+\delta^{c} d^{c}\right)=\left(d^{c}-\delta^{c}\right)^{2}=-\left(d^{c}+\delta^{c}\right)^{2} .
$$




\section{Discrete Dirac-Kähler and Hestenes Equations}

Let us introduce a discrete inhomogeneous form as follows

$$
\Omega=\sum_{r=0}^{4} \stackrel{r}{\omega},
$$

where $\stackrel{r}{\omega} \in K^{r}(4)$. A discrete analog of the Dirac-Kähler equation (1.2) is defined to be

$$
i\left(d^{c}+\delta^{c}\right) \Omega=m \Omega,
$$

where $i$ is the usual complex unit and $m$ is a positive number (mass parameter). It is clear that we can write this equation more explicitly by separating its homogeneous components as the system (1.3). Moreover, by (2.7)-(2.9) and (2.17)-(2.20), this system of equations for each $k=\left(k_{0}, k_{1}, k_{2}, k_{3}\right)$ can be expressed in terms of 16 difference equations as follows

$$
\begin{aligned}
i\left(\Delta_{0} \omega_{k}^{0}-\Delta_{1} \omega_{k}^{1}-\Delta_{2} \omega_{k}^{2}-\Delta_{3} \omega_{k}^{3}\right) & =m \omega_{k}^{0} \\
i\left(\Delta_{0} \omega_{k}+\Delta_{1} \omega_{k}^{01}+\Delta_{2} \omega_{k}^{02}+\Delta_{3} \omega_{k}^{03}\right) & =m \omega_{k}^{0} \\
i\left(\Delta_{1} \omega_{k}+\Delta_{0} \omega_{k}^{01}+\Delta_{2} \omega_{k}^{12}+\Delta_{3} \omega_{k}^{13}\right) & =m \omega_{k}^{1} \\
i\left(\Delta_{2} \omega_{k}+\Delta_{0} \omega_{k}^{02}-\Delta_{1} \omega_{k}^{12}+\Delta_{3} \omega_{k}^{23}\right) & =m \omega_{k}^{2} \\
i\left(\Delta_{3} \omega_{k}+\Delta_{0} \omega_{k}^{03}-\Delta_{1} \omega_{k}^{13}-\Delta_{2} \omega_{k}^{23}\right) & =m \omega_{k}^{3} \\
i\left(\Delta_{0} \omega_{k}^{1}-\Delta_{1} \omega_{k}^{0}-\Delta_{2} \omega_{k}^{012}-\Delta_{3} \omega_{k}^{013}\right) & =m \omega_{k}^{01} \\
i\left(\Delta_{0} \omega_{k}^{2}-\Delta_{2} \omega_{k}^{0}+\Delta_{1} \omega_{k}^{012}-\Delta_{3} \omega_{k}^{023}\right) & =m \omega_{k}^{02} \\
i\left(\Delta_{0} \omega_{k}^{3}-\Delta_{3} \omega_{k}^{0}+\Delta_{1} \omega_{k}^{013}+\Delta_{2} \omega_{k}^{023}\right) & =m \omega_{k}^{03} \\
i\left(\Delta_{1} \omega_{k}^{2}-\Delta_{2} \omega_{k}^{1}+\Delta_{0} \omega_{k}^{012}-\Delta_{3} \omega_{k}^{123}\right) & =m \omega_{k}^{12} \\
i\left(\Delta_{1} \omega_{k}^{3}-\Delta_{3} \omega_{k}^{1}+\Delta_{0} \omega_{k}^{013}+\Delta_{2} \omega_{k}^{123}\right) & =m \omega_{k}^{13} \\
i\left(\Delta_{2} \omega_{k}^{3}-\Delta_{3} \omega_{k}^{2}+\Delta_{0} \omega_{k}^{023}-\Delta_{1} \omega_{k}^{123}\right) & =m \omega_{k}^{23} \\
i\left(\Delta_{0} \omega_{k}^{12}-\Delta_{1} \omega_{k}^{02}+\Delta_{2} \omega_{k}^{01}+\Delta_{3} \omega_{k}\right) & =m \omega_{k}^{012} \\
i\left(\Delta_{0} \omega_{k}^{13}-\Delta_{1} \omega_{k}^{03}+\Delta_{3} \omega_{k}^{01}-\Delta_{2} \omega_{k}\right) & =m \omega_{k}^{013} \\
i\left(\Delta_{0} \omega_{k}^{23}-\Delta_{2} \omega_{k}^{03}+\Delta_{3} \omega_{k}^{02}+\Delta_{1} \omega_{k}\right) & =m \omega_{k}^{023} \\
i\left(\Delta_{1} \omega_{k}^{23}-\Delta_{2} \omega_{k}^{13}+\Delta_{3} \omega_{k}^{12}+\Delta_{0} \omega_{k}\right) & =m \omega_{k}^{123} \\
\left.i \omega_{k}^{123}-\Delta_{1} \omega_{k}^{023}+\Delta_{2} \omega_{k}^{013}-\Delta_{3} \omega_{k}^{012}\right) & =m \omega_{k}^{4}
\end{aligned}
$$

Let us define the Clifford multiplication in $K(4)$ by the following rules:

(a) $\quad x^{k} x^{k}=x^{k}, \quad x^{k} e_{\mu}^{k}=e_{\mu}^{k} x^{k}=e_{\mu}^{k}$,

(b) $e_{\mu}^{k} e_{\nu}^{k}+e_{\nu}^{k} e_{\mu}^{k}=2 g_{\mu \nu} x^{k}, \quad g_{\mu \nu}=\operatorname{diag}(1,-1,-1,-1)$,

(c) $e_{\mu_{1}}^{k} \cdots e_{\mu_{s}}^{k}=e_{\mu_{1} \cdots \mu_{s}}^{k}$ for $\quad 0 \leq \mu_{1}<\cdots<\mu_{s} \leq 3$, 
supposing the product to be zero in all other cases.

The operation is linearly extended to arbitrary discrete forms. For example, for $\stackrel{1}{\omega}, \stackrel{1}{\varphi} \in K^{1}(4)$ we have

$$
\begin{aligned}
\stackrel{1}{\omega} \stackrel{1}{\varphi}= & \left(\sum_{k} \sum_{\mu=0}^{3} \omega_{k}^{\mu} e_{\mu}^{k}\right)\left(\sum_{k} \sum_{\mu=0}^{3} \varphi_{k}^{\mu} e_{\mu}^{k}\right)=\sum_{k}\left(\omega_{k}^{0} \varphi_{k}^{0}-\omega_{k}^{1} \varphi_{k}^{1}-\omega_{k}^{2} \varphi_{k}^{2}-\omega_{k}^{3} \varphi_{k}^{3}\right) x^{k} \\
& +\sum_{k}\left[\left(\omega_{k}^{0} \varphi_{k}^{1}-\omega_{k}^{1} \varphi_{k}^{0}\right) e_{01}^{k}+\left(\omega_{k}^{0} \varphi_{k}^{2}-\omega_{k}^{2} \varphi_{k}^{0}\right) e_{02}^{k}+\left(\omega_{k}^{0} \varphi_{k}^{3}-\omega_{k}^{3} \varphi_{k}^{0}\right) e_{03}^{k}\right. \\
& \left.+\left(\omega_{k}^{1} \varphi_{k}^{2}-\omega_{k}^{2} \varphi_{k}^{1}\right) e_{12}^{k}+\left(\omega_{k}^{1} \varphi_{k}^{3}-\omega_{k}^{3} \varphi_{k}^{1}\right) e_{13}^{k}+\left(\omega_{k}^{2} \varphi_{k}^{3}-\omega_{k}^{3} \varphi_{k}^{2}\right) e_{23}^{k}\right] .
\end{aligned}
$$

Consider the following unit forms

$$
x=\sum_{k} x^{k}, \quad e=\sum_{k} e^{k}, \quad e_{\mu}=\sum_{k} e_{\mu}^{k}, \quad e_{\mu \nu}=\sum_{k} e_{\mu \nu}^{k} .
$$

Note that the unit 0 -form $x$ plays a role of the unit element in $K(4)$, i.e., for any $r$-form $\stackrel{r}{\omega}$ we have

$$
x \stackrel{r}{\omega}=\stackrel{r}{\omega} x=\stackrel{r}{\omega} .
$$

Proposition 3.1. The following holds:

$$
e_{\mu} e_{\nu}+e_{\nu} e_{\mu}=2 g_{\mu \nu} x, \quad \mu, \nu=0,1,2,3 .
$$

Proof. By the rule $e_{\mu}^{k} e_{\nu}^{k}+e_{\nu}^{k} e_{\mu}^{k}=2 g_{\mu \nu} x^{k}$ it is obvious.

The following proposition was proven in [15, Proposition 1].

Proposition 3.2. For any inhomogeneous form $\Omega \in K(4)$ we have

$$
\left(d^{c}+\delta^{c}\right) \Omega=\sum_{\mu=0}^{3} e_{\mu} \Delta_{\mu} \Omega,
$$

where $\Delta_{\mu}$ is the difference operator which acts on each component of $\Omega$ by the rule (2.6).

Clearly, the discrete Dirac-Kähler equation can be rewritten in the form

$$
i \sum_{\mu=0}^{3} e_{\mu} \Delta_{\mu} \Omega=m \Omega .
$$

Let $\Omega^{e v} \in K^{e v}(4)$ be a real-valued even inhomogeneous form, i.e., $\Omega^{e v}=$ $\stackrel{0}{\omega}+\stackrel{2}{\omega}+\stackrel{4}{\omega}$. A discrete analogue of the Hestenes equation (1.6) is defined by

$$
-\left(d^{c}+\delta^{c}\right) \Omega^{e v} e_{1} e_{2}=m \Omega^{e v} e_{0},
$$

where $e_{0}, e_{1}, e_{2}$ are given by (3.2). From (3.3) it follows that Eq. (3.4) is equivalent to

$$
-\sum_{\mu=0}^{3}\left(e_{\mu} \Delta_{\mu} \Omega^{e v}\right) e_{1} e_{2}=m \Omega^{e v} e_{0}
$$


This equation can be expressed in terms of difference equations as

$$
\begin{aligned}
\Delta_{0} \omega_{k}^{12}-\Delta_{1} \omega_{k}^{02}+\Delta_{2} \omega_{k}^{01}+\Delta_{3}{ }^{4} \omega_{k} & =m \omega^{0} \omega_{k}, \\
\Delta_{2}{ }^{0} \omega_{k}+\Delta_{0} \omega_{k}^{02}-\Delta_{1} \omega_{k}^{12}+\Delta_{3} \omega_{k}^{23} & =m \omega_{k}^{01}, \\
-\Delta_{1}{ }^{0} \omega_{k}-\Delta_{0} \omega_{k}^{01}-\Delta_{2} \omega_{k}^{12}-\Delta_{3} \omega_{k}^{13} & =m \omega_{k}^{02}, \\
-\Delta_{1} \omega_{k}^{23}+\Delta_{2} \omega_{k}^{13}-\Delta_{3} \omega_{k}^{12}-\Delta_{0} \omega_{k} & =m \omega_{k}^{03}, \\
-\Delta_{0}{ }^{0} \omega_{k}-\Delta_{1} \omega_{k}^{01}-\Delta_{2} \omega_{k}^{02}-\Delta_{3} \omega_{k}^{03} & =m \omega_{k}^{12}, \\
-\Delta_{0} \omega_{k}^{23}+\Delta_{2} \omega_{k}^{03}-\Delta_{3} \omega_{k}^{02}-\Delta_{1}{ }^{4} \omega_{k} & =m \omega_{k}^{13}, \\
\Delta_{0} \omega_{k}^{13}-\Delta_{1} \omega_{k}^{03}+\Delta_{3} \omega_{k}^{01}-\Delta_{2} \omega_{k} & =m \omega_{k}^{23}, \\
\Delta_{3} \omega_{k}+\Delta_{0} \omega_{k}^{03}-\Delta_{1} \omega_{k}^{13}-\Delta_{2} \omega_{k}^{23} & =m \omega_{k}^{4} .
\end{aligned}
$$

In [15, Proposition 5], it is proven that by a solution of the discrete Dirac-Kähler equation four independent real-valued solutions of the discrete Hestenes equation (3.4) are constructed. This is a discrete version of the well-known result for corresponding continuum equations [2].

Remark 3.3. It should be noted that the complex $K(4)$ does not give an exact geometric counterpart of the Clifford algebra $\mathcal{C} \ell(1,3)$ but a deformation of it. This problem arises because the operators $d^{c}$ and $\delta^{c}$ contain only finite difference operators of the forward type. The fact that both forward and backward differences are needed on the lattice is well-known [11]. For a suitable algebraic structure for lattice problems that reduced to a Clifford algebra in the continuum limit, we refer to [16]. The only way to avoid the deformation gap is to switch to operators which contain both types of differences. This is the subject of current work in progress.

\section{Plane Wave Solutions}

Let us consider the real-valued forms

$$
\Psi^{ \pm}=\sum_{k} \Psi_{k}^{ \pm} x^{k}
$$

where

$$
\Psi_{k}^{ \pm}=\left(x \pm p_{0} e_{12}\right)^{k_{0}}\left(x \pm p_{1} e_{12}\right)^{k_{1}}\left(x \pm p_{2} e_{12}\right)^{k_{2}}\left(x \pm p_{3} e_{12}\right)^{k_{3}},
$$

and $p_{\mu} \in \mathbb{R}$. Recall that $e_{12}$ is the unit 2 -form given by $(3.2)$. It is easy to check that

$$
\Delta_{\mu} \Psi_{k}^{ \pm}= \pm p_{\mu} \Psi_{k}^{ \pm} e_{12}, \quad \mu=0,1,2,3
$$

Therefore

$$
\left(d^{c}+\delta^{c}\right) \Psi^{ \pm}=\sum_{\mu=0}^{3} e_{\mu} \Delta_{\mu} \Psi^{ \pm}= \pm \sum_{\mu=0}^{3} e_{\mu} p_{\mu} \Psi^{ \pm} e_{12}
$$


It should be noted that the components $\Psi_{k}^{ \pm}$can be represented as

$$
\Psi_{k}^{ \pm}=\sum_{k}\left(\psi_{k}^{ \pm} x+\phi_{k}^{ \pm} e_{12}\right)
$$

where

$$
\psi_{k}^{ \pm}=\psi_{k}^{ \pm}\left(p_{0}, p_{1}, p_{2}, p_{3}\right) \quad \text { and } \quad \phi_{k}^{ \pm}=\phi_{k}^{ \pm}\left(p_{0}, p_{1}, p_{2}, p_{3}\right) .
$$

Hence $\Psi^{ \pm}$are inhomogeneous real-valued even forms of the form

$$
\Psi^{ \pm}=\psi^{ \pm}+\phi^{ \pm}
$$

where

$$
\psi^{ \pm}=\sum_{k} \psi_{k}^{ \pm} x^{k}, \quad \phi^{ \pm}=\sum_{k} \phi_{k}^{ \pm} e_{12}^{k}
$$

We wish to find a solution of the discrete Hestenes equation (3.4) of the form

$$
\Omega^{ \pm}=A \Psi^{ \pm},
$$

where $A \in K^{e v}(4)$ is a constant real-valued form. Hence $A$ can be expanded as

$$
A=\alpha^{0} x+\sum_{\mu<\nu} \alpha^{\mu \nu} e_{\mu \nu}+\alpha^{4} e
$$

where $\alpha^{0}, \alpha^{\mu \nu}, \alpha^{4} \in \mathbb{R}$ and $x, e_{\mu \nu}, e$ are the unit forms given by (3.2). We have

$$
\begin{aligned}
\left(d^{c}+\delta^{c}\right) \Omega^{ \pm}= & \left(d^{c}+\delta^{c}\right) A \Psi^{ \pm}=\sum_{\mu=0}^{3} e_{\mu} \Delta_{\mu}\left(A \Psi^{ \pm}\right) \\
& =\sum_{\mu=0}^{3} e_{\mu} A\left(\Delta_{\mu} \Psi^{ \pm}\right)=\sum_{\mu=0}^{3} e_{\mu} A \sum_{k}\left(\Delta_{\mu} \Psi_{k}^{ \pm}\right) x^{k} \\
& = \pm \sum_{\mu=0}^{3} e_{\mu} p_{\mu} A \sum_{k} \Psi_{k}^{ \pm} x^{k} e_{12}= \pm \sum_{\mu=0}^{3} e_{\mu} p_{\mu} A \Psi^{ \pm} e_{12}
\end{aligned}
$$

Substituting this into the discrete Hestenes equation (3.4) we obtain

$$
\mp\left(\sum_{\mu=0}^{3} e_{\mu} p_{\mu} A \Psi^{ \pm} e_{12}\right) e_{12}=m A \Psi^{ \pm} e_{0} .
$$

By definition, $e_{12} e_{12}=-x$. This yields

$$
\pm \sum_{\mu=0}^{3} e_{\mu} p_{\mu} A \Psi^{ \pm}=m A \Psi^{ \pm} e_{0} .
$$

Since $\Psi^{ \pm} e_{0}=e_{0} \Psi^{ \pm}$, the equation above reduces to

$$
\sum_{\mu=0}^{3} e_{\mu} p_{\mu} A=m A e_{0}
$$

in the case of the form $\Psi^{+}$and to

$$
-\sum_{\mu=0}^{3} e_{\mu} p_{\mu} A=m A e_{0}
$$


in the case of the form $\Psi^{-}$. Firstly, we take the form $\Psi^{-}$. Equation (4.8) implies

$$
-\left(p_{0} x+p_{1} e_{0} e_{1}+p_{2} e_{0} e_{2}+p_{3} e_{0} e_{3}\right) A=m e_{0} A e_{0},
$$

since $e_{0} e_{0}=x$. By trivial computation one finds that

$$
\left(p_{0} x-\sum_{\mu=1}^{3} p_{\mu} e_{0} e_{\mu}\right)\left(p_{0} x+\sum_{\mu=1}^{3} p_{\mu} e_{0} e_{\mu}\right)=\left(p_{0}^{2}-\sum_{\mu=1}^{3} p_{\mu}^{2}\right) x .
$$

Then multiplying both sides of Eq. (4.9) by $-\left(p_{0} x-p_{1} e_{0} e_{1}-p_{2} e_{0} e_{2}-p_{3} e_{0} e_{3}\right)$ we obtain

$$
\left(p_{0}^{2}-\sum_{\mu=1}^{3} p_{\mu}^{2}\right) x A=-m\left(p_{0} x-\sum_{\mu=1}^{3} p_{\mu} e_{0} e_{\mu}\right) e_{0} A e_{0},
$$

or equivalently,

$$
\left(p_{0}^{2}-\sum_{\mu=1}^{3} p_{\mu}^{2}\right) A=-m\left(\sum_{\mu=0}^{3} e_{\mu} p_{\mu} A\right) e_{0} .
$$

Applying (4.8) gives

$$
\left(p_{0}^{2}-\sum_{\mu=1}^{3} p_{\mu}^{2}\right) A=m^{2} A e_{0} e_{0}
$$

or equivalently,

$$
\left(p_{0}^{2}-\sum_{\mu=1}^{3} p_{\mu}^{2}\right) A=m^{2} A .
$$

Thus Eq. (4.8) has a non-trivial solution if and only if

$$
p_{0}^{2}-p_{1}^{2}-p_{2}^{2}-p_{3}^{2}=m^{2},
$$

or

$$
p_{0}= \pm \sqrt{m^{2}+p_{1}^{2}+p_{2}^{2}+p_{3}^{2}} .
$$

Proposition 4.1. The form $A \Psi^{-}$is a non-trivial solution of the discrete Hestenes equation (3.4) if and only if the condition (4.10) holds.

It is clear that the same is true for $A \Psi^{+}$in place $A \Psi^{-}$, i.e., if we take Eq. (4.7) we obtain the condition (4.10) again.

Let $p=\left\{p_{0}, p_{1}, p_{2}, p_{3}\right\}$ be the energy-momentum vector of a particle with (proper) mass $m$. Then the relation (4.10) is the energy-momentum relation. It is known that in the continuum theory the Hestenes equation (1.5) admits the plane wave solutions of the form

$$
\Phi^{ \pm}=A \exp \left( \pm \gamma_{2} \gamma_{1} p \cdot x\right) .
$$

Thus the forms $\Omega^{ \pm}=A \Psi^{ \pm}$are discrete versions of the plane wave solutions $\Phi^{ \pm}$.

Let us represent the even real-valued form (4.6) as

$$
A=A_{+}+A_{-},
$$


where

$$
\begin{aligned}
& A_{+}=\alpha^{0} x+\alpha^{12} e_{12}+\alpha^{13} e_{13}+\alpha^{23} e_{23}, \\
& A_{-}=\alpha^{01} e_{01}+\alpha^{02} e_{02}+\alpha^{03} e_{03}+\alpha^{4} e .
\end{aligned}
$$

It is easy to check that $A_{+}$commutes with $e_{0}$ and $A_{-}$anticommutes with it, i.e.,

$$
e_{0} A_{ \pm}= \pm A_{ \pm} e_{0}
$$

Lemma 4.2. The form $e_{0 \mu} A_{-}$commutes with $e_{0}$ and has the view (4.11), while $e_{0 \mu} A_{+}$anticommutes with $e_{0}$ and has the view (4.12) for any $\mu=1,2,3$.

Proof. For $\mu=1$ we have

$$
\begin{aligned}
e_{01} A_{-} & =e_{01}\left(\alpha^{01} e_{01}+\alpha^{02} e_{02}+\alpha^{03} e_{03}+\alpha^{4} e\right) \\
& =\alpha^{01} x-\alpha^{02} e_{12}-\alpha^{03} e_{13}+\alpha^{4} e_{23}
\end{aligned}
$$

and

$$
\begin{aligned}
e_{01} A_{+} & =e_{01}\left(\alpha^{0} x+\alpha^{12} e_{12}+\alpha^{13} e_{13}+\alpha^{23} e_{23}\right) \\
& =\alpha^{0} e_{01}-\alpha^{12} e_{02}-\alpha^{13} e_{03}+\alpha^{23} e .
\end{aligned}
$$

The same proof remains valid for all other cases.

Theorem 4.3. The forms $\Omega^{ \pm}=A \Psi^{ \pm}$are non-trivial solutions of the discrete Hestenes equation if and only if the conditions

$$
A_{ \pm}=\frac{p_{1} e_{01}+p_{2} e_{02}+p_{3} e_{03}}{m-p_{0}} A_{\mp}
$$

hold, or equivalently,

$$
A_{\mp}=-\frac{p_{1} e_{01}+p_{2} e_{02}+p_{3} e_{03}}{m+p_{0}} A_{ \pm} .
$$

Proof. Let $A \Psi^{-}$satisfy Eq. (3.4). Then $A=A_{+}+A_{-}$satisfies Eq. (4.8):

$$
-\left(\sum_{\mu=0}^{3} e_{\mu} p_{\mu}\right)\left(A_{+}+A_{-}\right)=m\left(A_{+}+A_{-}\right) e_{0} .
$$

From this we obtain

$$
-\left(\sum_{\mu=1}^{3} e_{0} e_{\mu} p_{\mu}\right)\left(A_{+}+A_{-}\right)=p_{0}\left(A_{+}+A_{-}\right)+m e_{0}\left(A_{+}+A_{-}\right) e_{0} .
$$

Applying (4.13) we can rewrite the above relationship as

$$
-\left(\sum_{\mu=1}^{3} e_{0} e_{\mu} p_{\mu}\right)\left(A_{+}+A_{-}\right)=\left(p_{0}+m\right) A_{+}+\left(p_{0}-m\right) A_{-} .
$$

By Lemma 4.2, collecting like terms gives

$$
\begin{aligned}
& -\left(e_{0} e_{1} p_{1}+e_{0} e_{2} p_{2}+e_{0} e_{3} p_{3}\right) A_{+}=\left(p_{0}-m\right) A_{-}, \\
& -\left(e_{0} e_{1} p_{1}+e_{0} e_{2} p_{2}+e_{0} e_{3} p_{3}\right) A_{-}=\left(p_{0}+m\right) A_{+} .
\end{aligned}
$$

Conversely, substituting (4.14) into (4.15) yields the condition (4.10). It follows that $A \Psi^{-}$is a non-trivial solution of Eq. (3.4).

The same is true for $A \Psi^{+}$in place $A \Psi^{-}$. 
It is clear that the conditions (4.14) and (4.15) can be rewritten as systems of four linear algebraic equations. For example, from (4.16) we obtain

$$
\begin{aligned}
& \left(m-p_{0}\right) \alpha^{01}-p_{1} \alpha^{0}-p_{2} \alpha^{12}-p_{3} \alpha^{13}=0, \\
& \left(m-p_{0}\right) \alpha^{02}-p_{2} \alpha^{0}+p_{1} \alpha^{12}-p_{3} \alpha^{23}=0, \\
& \left(m-p_{0}\right) \alpha^{03}-p_{3} \alpha^{0}+p_{1} \alpha^{13}+p_{2} \alpha^{23}=0, \\
& \left(m-p_{0}\right) \alpha^{4}-p_{3} \alpha^{12}+p_{2} \alpha^{13}-p_{1} \alpha^{23}=0 .
\end{aligned}
$$

From this the form (4.12) can be written as

$$
\begin{aligned}
A_{-}= & \frac{p_{1} \alpha^{0}+p_{2} \alpha^{12}+p_{3} \alpha^{13}}{m-p_{0}} e_{01}+\frac{p_{2} \alpha^{0}-p_{1} \alpha^{12}+p_{3} \alpha^{23}}{m-p_{0}} e_{02} \\
& +\frac{p_{3} \alpha^{0}-p_{1} \alpha^{13}-p_{2} \alpha^{23}}{m-p_{0}} e_{03}+\frac{p_{3} \alpha^{12}-p_{2} \alpha^{13}+p_{1} \alpha^{23}}{m-p_{0}} e .
\end{aligned}
$$

Hence

$$
\begin{aligned}
A= & a_{1}\left(\left(m-p_{0}\right) x+p_{1} e_{01}+p_{2} e_{02}+p_{3} e_{03}\right) \\
& +a_{2}\left(\left(m-p_{0}\right) e_{12}+p_{2} e_{01}-p_{1} e_{02}+p_{3} e\right) \\
& +a_{3}\left(\left(m-p_{0}\right) e_{13}+p_{3} e_{01}-p_{1} e_{03}-p_{2} e\right) \\
& +a_{4}\left(\left(m-p_{0}\right) e_{23}+p_{3} e_{02}-p_{2} e_{03}+p_{1} e\right)
\end{aligned}
$$

where

$$
a_{1}=\frac{\alpha^{0}}{m-p_{0}}, \quad a_{2}=\frac{\alpha^{12}}{m-p_{0}}, \quad a_{3}=\frac{\alpha^{13}}{m-p_{0}}, \quad a_{4}=\frac{\alpha^{23}}{m-p_{0}} .
$$

Thus the general plane wave solution of Eq. (3.4) is $\Omega^{-}=A \Psi^{-}$, where $A$ is given by (4.18). Note that for given $p_{\mu}, \mu=1,2,3$, there are four linearly independent solutions of the form (4.5) for each positive and negative $p_{0}$.

Similarly, from (4.17) we obtain

$$
\begin{aligned}
& \left(m+p_{0}\right) \alpha^{0}+p_{1} \alpha^{01}+p_{2} \alpha^{02}+p_{3} \alpha^{03}=0, \\
& \left(m+p_{0}\right) \alpha^{12}-p_{1} \alpha^{02}+p_{2} \alpha^{01}+p_{3} \alpha^{4}=0, \\
& \left(m+p_{0}\right) \alpha^{13}+p_{3} \alpha^{01}-p_{1} \alpha^{03}-p_{2} \alpha^{4}=0, \\
& \left(m+p_{0}\right) \alpha^{23}+p_{3} \alpha^{02}-p_{2} \alpha^{03}+p_{1} \alpha^{4}=0 .
\end{aligned}
$$

This leads to

$$
\begin{aligned}
A= & b_{1}\left(\left(m+p_{0}\right) e_{01}-p_{1} x-p_{2} e_{12}-p_{3} e_{13}\right) \\
& +b_{2}\left(\left(m+p_{0}\right) e_{02}-p_{2} x+p_{1} e_{12}-p_{3} e_{23}\right) \\
& +b_{3}\left(\left(m+p_{0}\right) e_{03}-p_{3} x+p_{1} e_{13}+p_{2} e_{23}\right) \\
& +b_{4}\left(\left(m+p_{0}\right) e-p_{3} e_{12}+p_{2} e_{13}-p_{1} e_{23}\right),
\end{aligned}
$$

where

$$
b_{1}=\frac{\alpha^{01}}{m+p_{0}}, \quad b_{2}=\frac{\alpha^{02}}{m+p_{0}}, \quad b_{3}=\frac{\alpha^{03}}{m+p_{0}}, \quad b_{4}=\frac{\alpha^{4}}{m+p_{0}} .
$$


By Theorem 4.3, (4.19) is equivalent to (4.18).

In the same way, the explicit formula for $A$ can be written in the case of the plane wave solution $\Omega^{+}=A \Psi^{+}$.

\section{Acknowledgements}

The author would like to thank N. Faustino for valuable discussions. The author also thanks the referees for careful reviews and for fruitful suggestions.

Open Access. This article is distributed under the terms of the Creative Commons Attribution 4.0 International License (http://creativecommons.org/licenses/by/4. $0 /$ ), which permits unrestricted use, distribution, and reproduction in any medium, provided you give appropriate credit to the original author(s) and the source, provide a link to the Creative Commons license, and indicate if changes were made.

\section{References}

[1] Baylis, W.E. (ed.): Clifford (Geometric) Algebra with Applications to Physics, Mathematics, and Engineering. Birkhäuser, Boston (1996)

[2] Baylis, W.E.: Comment on 'Dirac theory in spacetime algebra'. J. Phys. A Math. Gen. 35, 4791-4796 (2002)

[3] Dezin, A.A.: Multidimensional analysis and discrete models. CRC Press, Boca Raton (1995)

[4] Faustino, N., Kähler, U., Sommen, F.: Discrete Dirac operators in Clifford analysis. Adv. Appl. Cliff. Alg. 17(3), 451-467 (2007)

[5] Faustino, N.: Solutions for the Klein-Gordon and Dirac equations on the lattice based on Chebyshev polynomials. Complex Anal. Oper. Theory 10(2), 379-399 (2016)

[6] Faustino, N.: A conformal group approach to the Dirac-Kahler system on the lattice. Math. Methods Appl. Sci. 40(11), 4118-4127 (2017)

[7] Hestenes, D.: Real Spinor Fields. J. Math. Phys. 8(4), 798-808 (1967)

[8] Hestenes, D.: Spacetime algebra. Gordon and Breach, New York (1966)

[9] Kähler, E.: Der innere differentialkül. Rendiconti di Matematica e delle sue Applicazioni 21(3-4), 425-523 (1962)

[10] Kanamori, I., Kawamoto, N.: Dirac-Kähler fermion from Clifford product with noncommutative differential form on a lattice. Int. J. Mod. Phys. A 19(5), 695-736 (2004)

[11] Rabin, J.M.: Homology theory of lattice fermion doubling. Nucl. Phys. B 201(2), 315-332 (1982)

[12] Sushch, V.: A discrete model of the Dirac-Kähler equation. Rep. Math. Phys. 73(1), 109-125 (2014)

[13] Sushch, V.: On the chirality of a discrete Dirac-Kähler equation. Rep. Math. Phys. 76(2), 179-196 (2015)

[14] Sushch, V.: Discrete Dirac-Kähler equation and its formulation in algebraic form. Pliska Stud. Math. 26, 225-238 (2016)

[15] Sushch, V.: Discrete Dirac-Kähler and Hestenes equations. In: Pinelas, S. et al. (eds.) Differential and difference equations with applications. ICDDEA, 
Amadora, Portugal, May 2015, Selected Contributions. Springer Proceedings in Mathematics and Statistics, vol. 164, pp. 433-442. Springer, Cham (2016)

[16] Vaz, J.: Clifford-like Calculus over Lattice. Adv. Appl. Clifford Alg. 7(1), 37-70 (1997)

Volodymyr Sushch

Koszalin University of Technology

Sniadeckich 2

75-453 Koszalin

Poland

e-mail: volodymyr.sushch@tu.koszalin.pl

Received: January 29, 2018.

Accepted: June 25, 2018. 